Supporting Information

\title{
Intertwined Nanosponge Solid-State Polymer Electrolyte for Rollable and Foldable Lithium-Ion Batteries
}

\author{
Saewoong Oh${ }^{1}$, Van Hiep Nguyen ${ }^{1}$, Van-Tien Bui ${ }^{1}$, Sanghee Nam ${ }^{1}$, Manmatha Mahato ${ }^{1}$, \\ and Il-Kwon $\mathrm{Oh}^{1, *}$
}

1 National Creative Research Initiative for Functionally Antagonistic Nano-Engineering,

Department of Mechanical Engineering, Korea Advanced Institute of Science and Technology (KAIST), 291 Daehak-ro, Yuseong-gu, Daejeon 34141, Republic of Korea.

${ }^{*}$ Corresponding author

e-mail: $\underline{\text { kkoh@kaist.ac.kr }}$ 


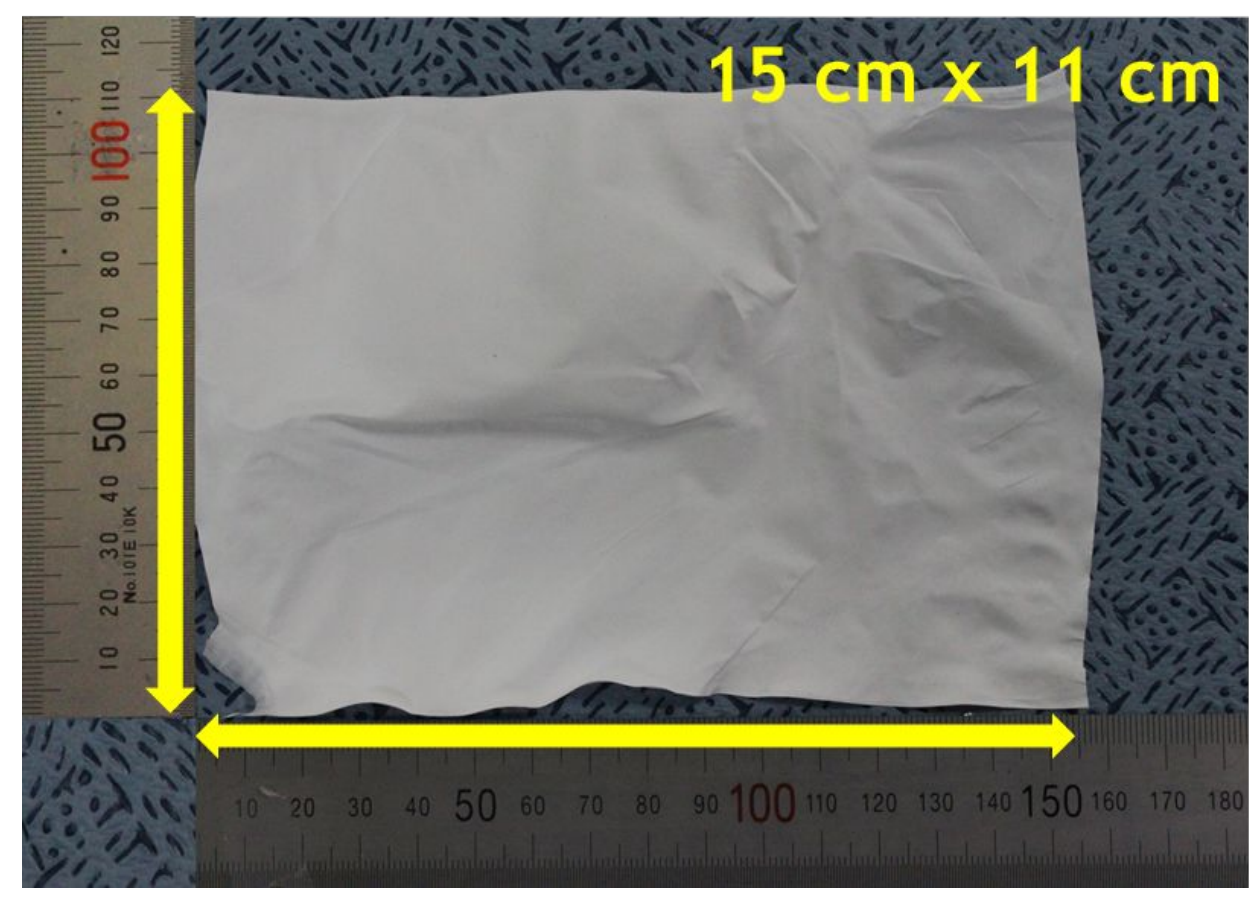

Figure S1. Optical image of IN in $15 \mathrm{~cm} \times 11 \mathrm{~cm}$ scale 

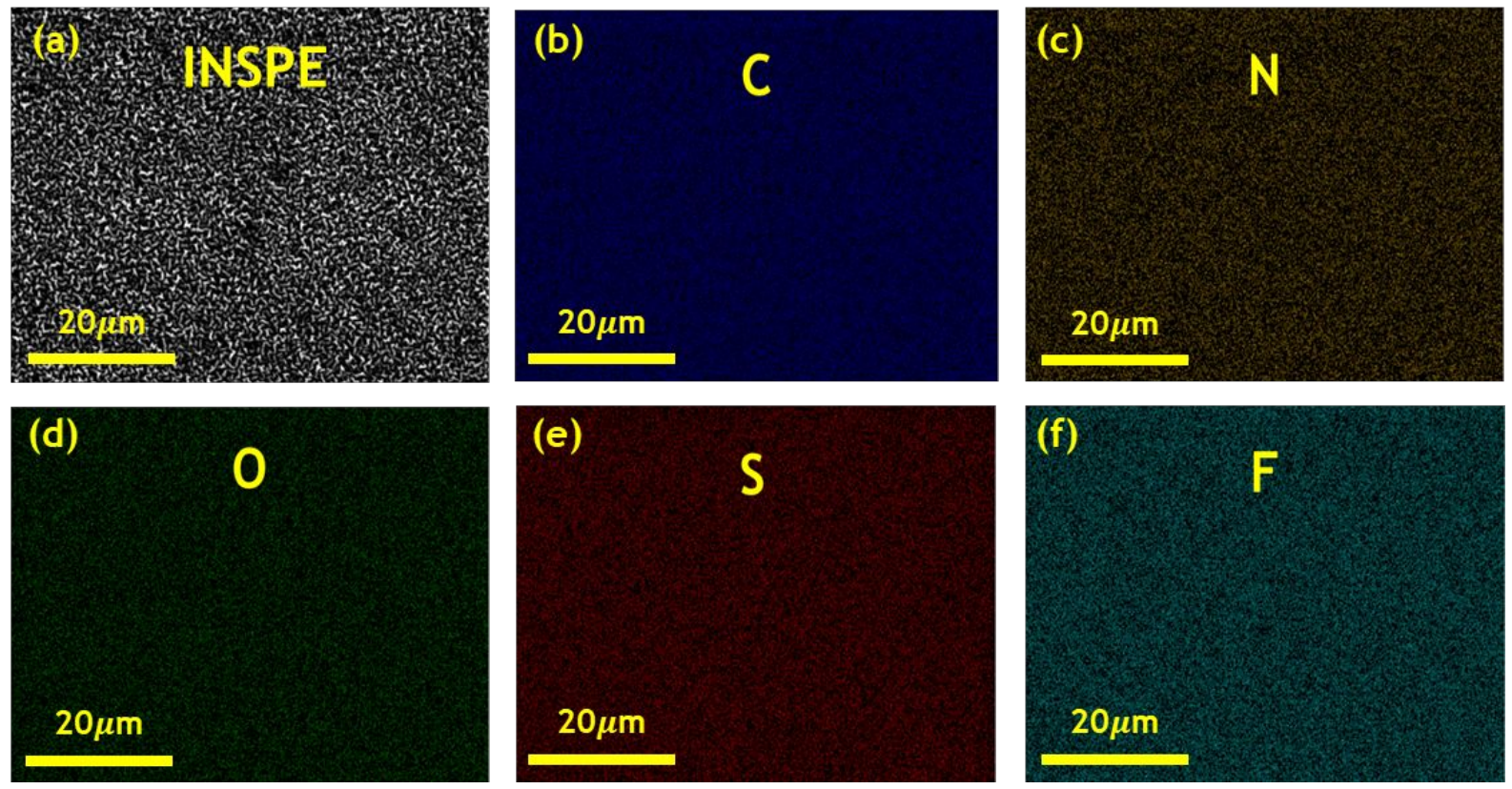

Figure S2. a) Surface SEM images of INSPEs and corresponding EDX mapping results for b)

C element, c) $\mathrm{N}$ element, d) O element, e) S element, and f) F element. 

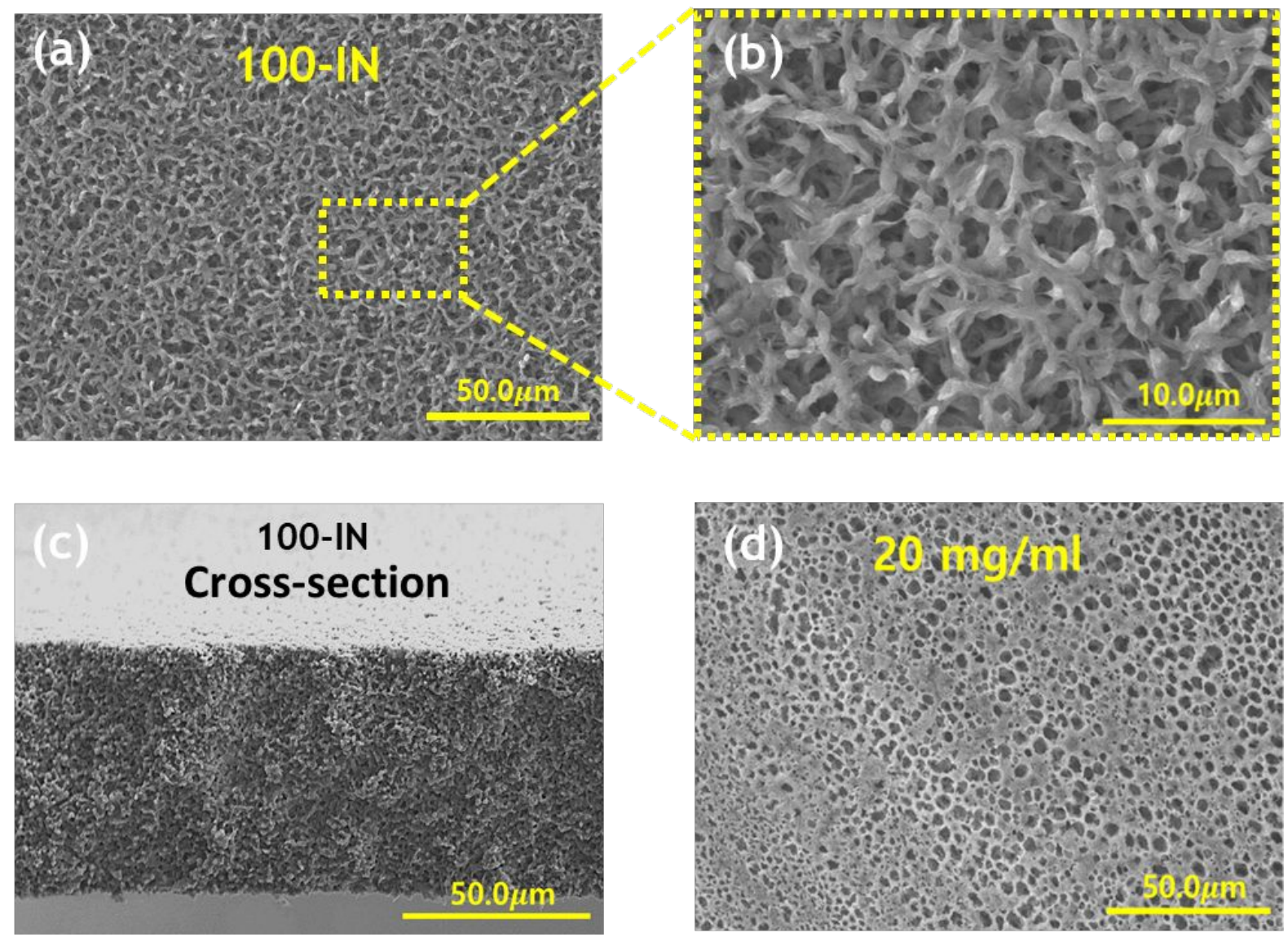

Figure S3. a) Surface SEM image of 10-IN and b) its magnified SEM image; c) Crosssectional SEM image of 100-IN; d) Surface SEM image of 20-IN 
(a)

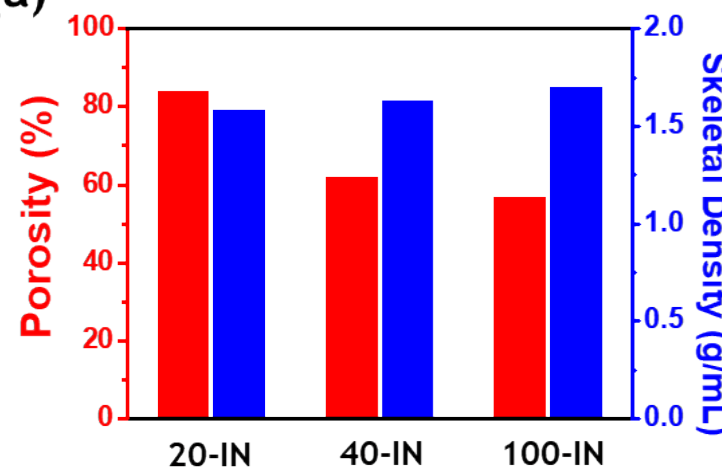

(c)

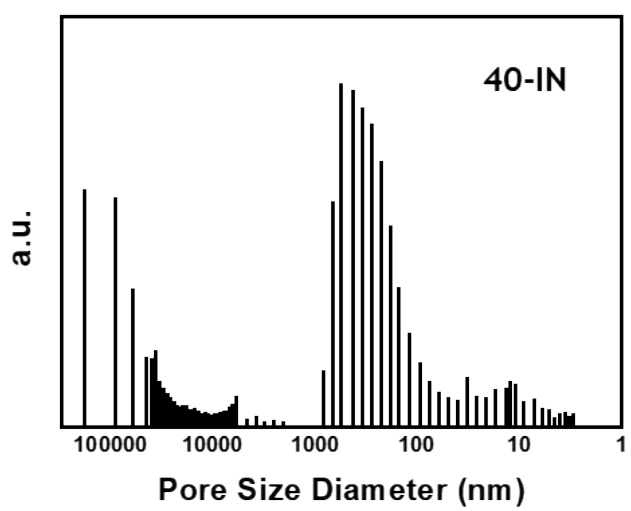

(b)

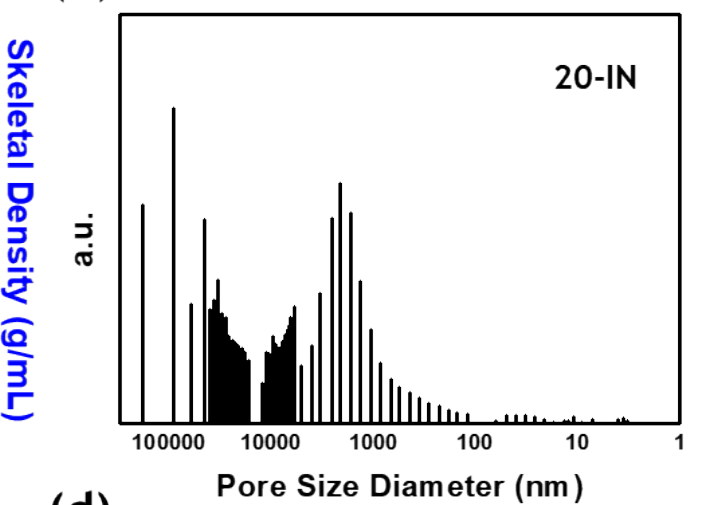

(d)

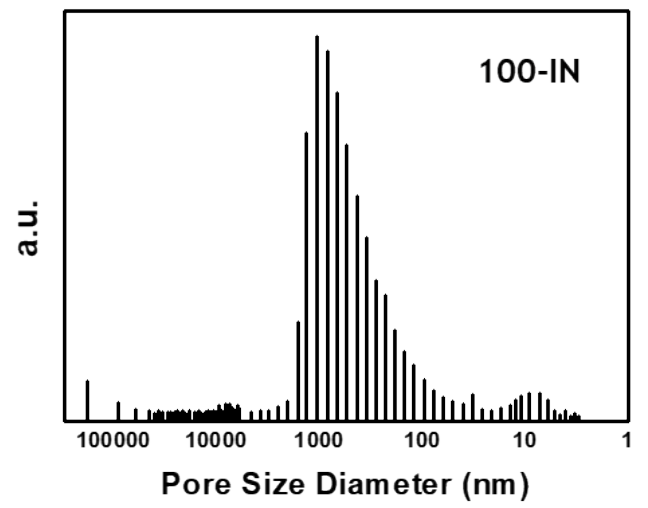

Figure S4. a) Porosity (red) and skeletal density (blue) of 20-IN, 40-IN, 100-IN; b) Pore size distribution of 20-IN; c) Pore size distribution of 40-IN; d) Pore size distribution of 100-IN; 

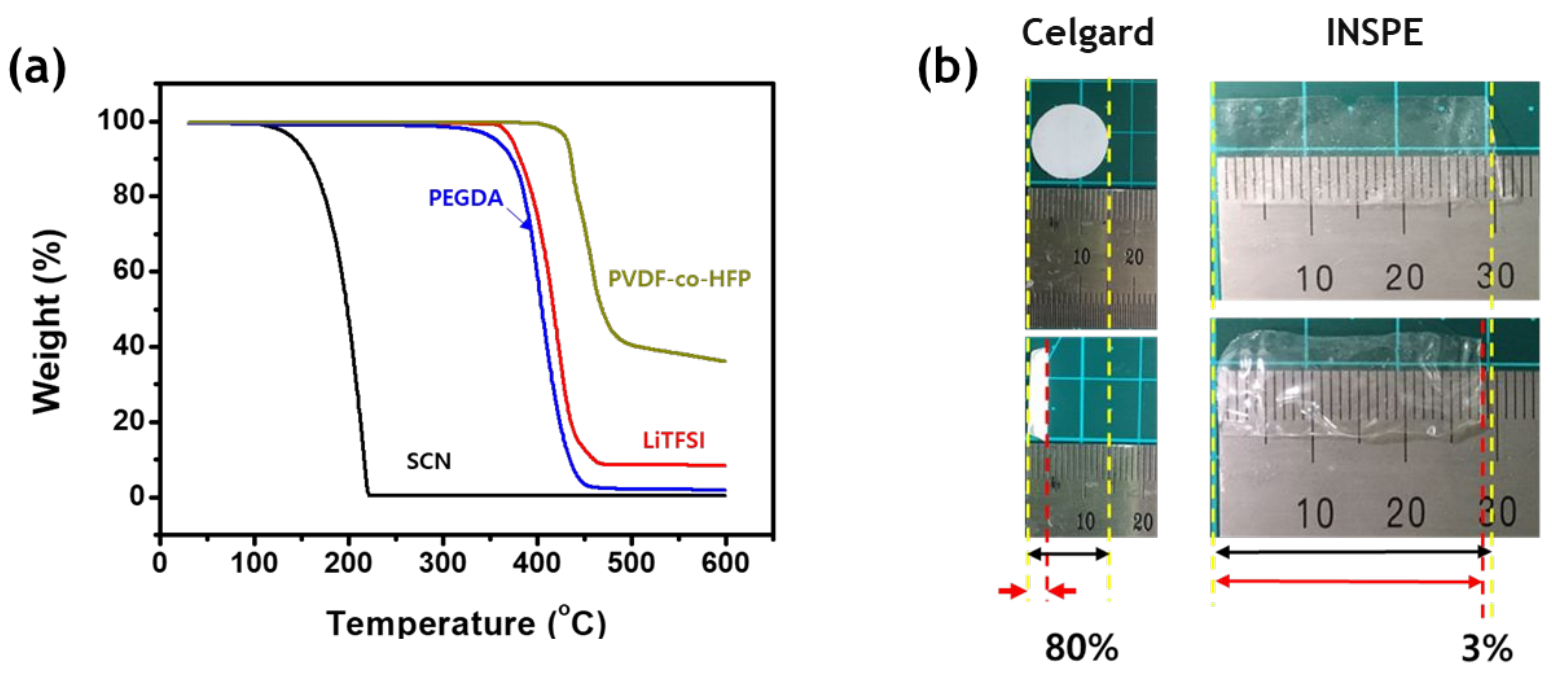

Figure S5. a) TGA thermograms of neat SCN, PEGDA, LiTFSI, and PVDF-co-HFP; b) Optical images of comparing commercial separator (Celgard) and as-fabricated INSPE after placing them at $90^{\circ} \mathrm{C}$ for $30 \mathrm{~min}$. 
(a)

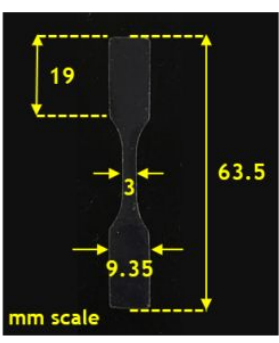

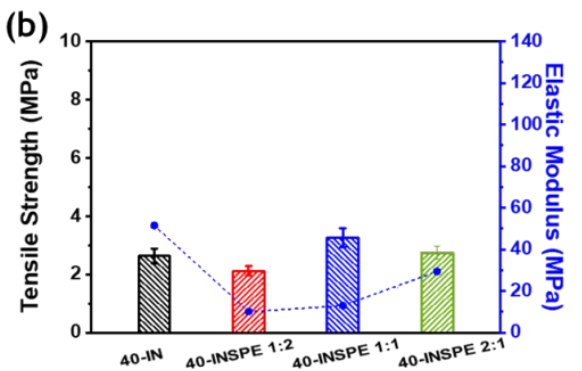

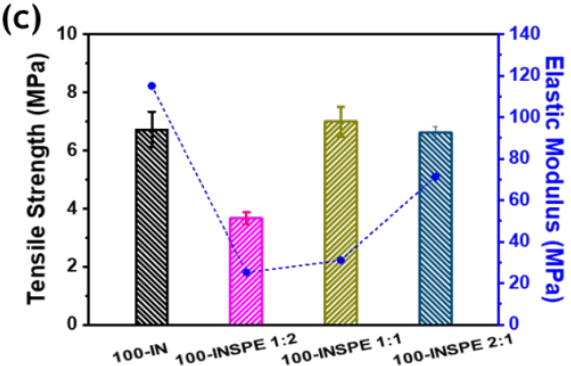

Figure S6. a) Dog-bone shape and its dimension for tensile test specimen; b) Ultimate tensile strength and elastic modulus of 40-IN, 40-INSPE 1:2, 40-INSPE1:1 and 40-INSPE 2:1; c) Ultimate tensile strength and elastic modulus of 100-IN, 100-INSPE 1:2, 100-INSPE 1:1, and 100-INSPE 2:1 
Table S1. Comparison of as-fabricated 40-IN and 100-IN with other PVDF-co-HFP based membranes.

\begin{tabular}{|c|c|c|}
\hline Membrane & $\begin{array}{c}\text { Ultimate Tensile } \\
\text { Strength } \\
\text { (MPa) }\end{array}$ & $\begin{array}{c}\text { Elongation } \\
\text { (\%) }\end{array}$ \\
\hline $\begin{array}{c}\text { 40-IN } \\
\text { (This work) }\end{array}$ & 2.5 & $\mathbf{5 3}$ \\
\hline $\begin{array}{c}\text { 100-IN } \\
\text { (This work) }\end{array}$ & 6.6 & 37 \\
\hline $\begin{array}{c}\text { PVDF-co-HFP } \\
\text { (solvent evaporation) }\end{array}$ & 6.06 & 100 \\
\hline $\begin{array}{c}\text { PVDF-co-HFP } \\
\text { (phase inversion) }\end{array}$ & 5.9 & 31 \\
\hline $\begin{array}{c}\text { Blending the EVOH-graft- } \\
\text { LiCPSI with the PVDF-HFP } \\
\text { (solution casting) }\end{array}$ & 8.2 & 78 \\
\hline $\begin{array}{c}\text { PVDF-co-HFP } \\
\text { (Electrospun) }\end{array}$ & 2.7 & \\
\hline
\end{tabular}


Table S2. Comparison of as-fabricated INSPEs with other PEO(DA)/SCN/LiTFSi based solid polymer electrolytes.

\begin{tabular}{|c|c|c|c|}
\hline Solid Polymer Electrolyte & $\begin{array}{c}\text { Ultimate Tensile } \\
\text { Strength } \\
\text { (MPa) } \\
\end{array}$ & $\begin{array}{c}\text { Elongation } \\
(\%)\end{array}$ & $\begin{array}{c}\text { Ionic } \\
\text { Conductivity } \\
\left(25^{\circ} \mathrm{C}\right)\left(\mathrm{S} \mathrm{cm}^{-1}\right) \\
\end{array}$ \\
\hline $\begin{array}{l}\text { 40-INSPE 1:2 } \\
\text { (This work) }\end{array}$ & 2.1 & 36.7 & $1.04 \times 10^{-3}$ \\
\hline $\begin{array}{l}\text { 100-INSPE 1:2 } \\
\text { (This work) }\end{array}$ & 3.6 & 46.2 & $5.62 \times 10^{-4}$ \\
\hline $\begin{array}{l}\text { 40-INSPE } 1: 1 \\
\text { (This work) }\end{array}$ & 3.2 & 28.6 & $1.27 \times 10^{-4}$ \\
\hline $\begin{array}{l}\text { 100-INSPE 1:1 } \\
\text { (This work) }\end{array}$ & 6.9 & 40.5 & $7.47 \times 10^{-5}$ \\
\hline $\begin{array}{l}\text { 40-INSPE 2:1 } \\
\text { (This work) }\end{array}$ & 2.7 & 11.6 & $5.53 \times 10^{-5}$ \\
\hline $\begin{array}{l}\text { 100-INSPE 2:1 } \\
\text { (This work) }\end{array}$ & 6.6 & 24.3 & $4.38 \times 10^{-5}$ \\
\hline $\begin{array}{c}\text { PEGDA700/SCN/LiTFSI } \\
(28 / 42 / 30)^{74}\end{array}$ & 0.11 & 5.9 & $0.58 \times 10^{-3}$ \\
\hline $\begin{array}{c}\text { PEGDA700/SCN/LiTFSI } \\
(35 / 35 / 30)^{74} \\
\end{array}$ & 0.2 & 7.7 & $0.52 \times 10^{-3}$ \\
\hline $\begin{array}{c}\text { TAME }_{n} \mathrm{PO} / \mathrm{TME}_{\mathrm{n}} \mathrm{PO} / \\
\mathrm{LiCF}_{3} \mathrm{SO}_{3}{ }^{75} \\
\end{array}$ & 1.2 & 2.5 & $2.0 \times 10^{-4}$ \\
\hline $\begin{array}{l}\text { PEGBCDMA/SCN/LiTFSI } \\
(20 / 40 / 40)^{76}\end{array}$ & 0.04 & 7.9 & $5.5 \times 10^{-4}$ \\
\hline $\begin{array}{c}\text { PEGDA/SCN/LiTFSI } \\
(20 / 40 / 40)^{76} \\
\end{array}$ & 0.24 & 27 & $9 \times 10^{-4}$ \\
\hline $\begin{array}{c}\text { PEGDA/SCN/LiTFSI } \\
(21 / 49 / 30)^{60} \\
\end{array}$ & 0.062 & 7 & $1 \times 10^{-3}$ \\
\hline $\begin{array}{c}\text { PEGDA/SCN/LiTFSI } \\
(28 / 42 / 30)^{60} \\
\end{array}$ & 0.095 & 4 & $6 \times 10^{-4}$ \\
\hline ETPTA/SCN/LiTFSI ${ }^{32}$ & 0.13 & 18 & $5 \times 10^{-3}$ \\
\hline $\begin{array}{l}\text { 1M LiTFSI in SCN + } \\
\text { PVDF membrane }\end{array}$ & 0.04 & 26 & $3.5 \times 10^{-3}$ \\
\hline
\end{tabular}


(a)

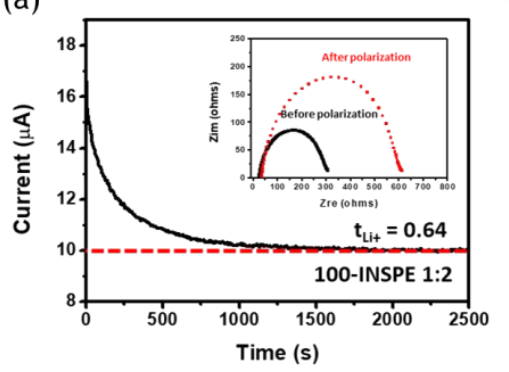

(b)

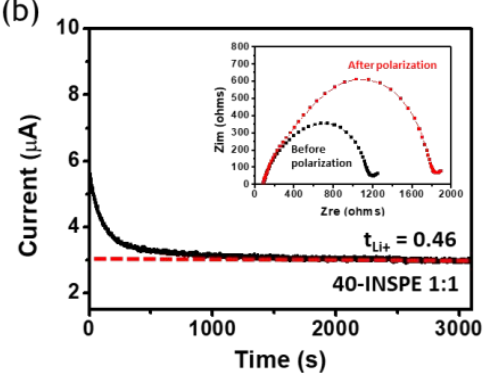

(c)

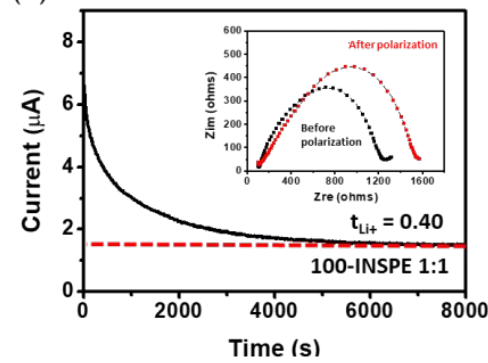

(d)

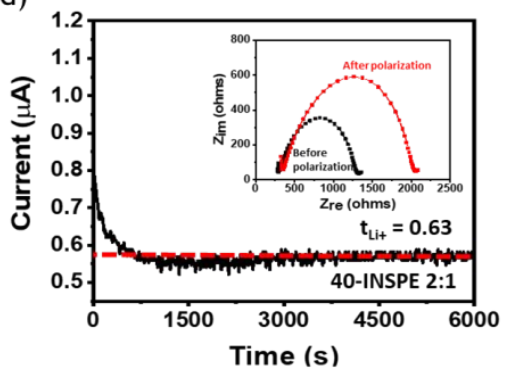

(e)

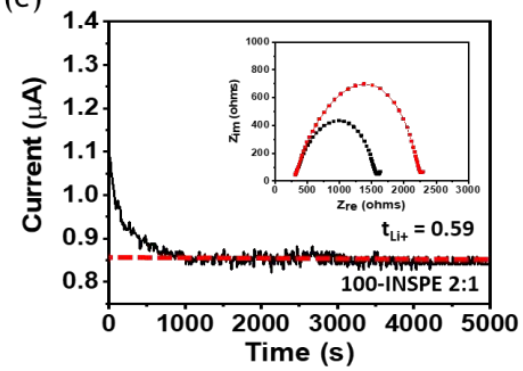

Figure S7. Chronoamperometry plot of a) Li/100-INSPE 1:2/Li, b) Li/40-INSPE 1:1/Li, c) Li/100-INSPE 1:1/Li, d) Li/40-INSPE 2:1/Li, and e) Li/100-INSPE 2:1/Li with bias potential of $10 \mathrm{mV}$ at $25^{\circ} \mathrm{C}$ along with corresponding EIS curves of before and after chronoamperometry test. 

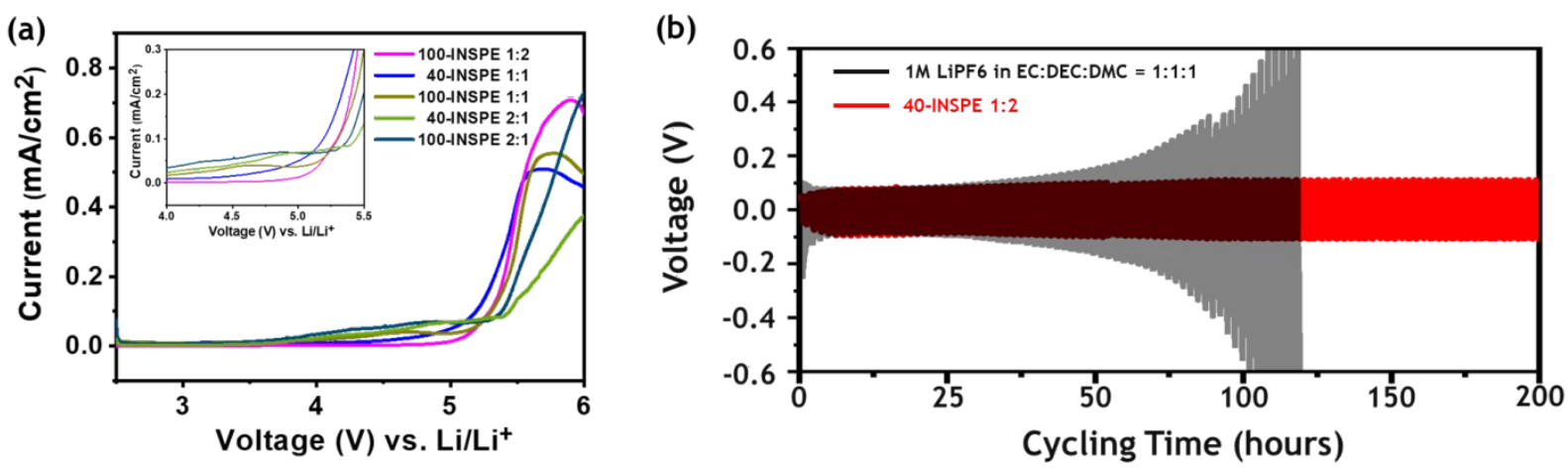

Figure S8. (a) LSV curve of 100-INSPE 1:2, 40-INSPE 1:1, 100-INSPE 1:1, 40-INSPE 2:1, and 100-INSPE 2:1 in the rage of $2.5 \mathrm{~V}$ to $6 \mathrm{~V}$ recorded at $10 \mathrm{mVs}^{-1}$. (b) Galvanostatic cycling profiles of $\mathrm{Li} /$ 40-INSPE 1:2 / Li and $\mathrm{Li}$ / commercial liquid electrolyte / Li symmetrical cells at 1mA $\mathrm{cm}^{-2}$ with cut-off capacity of $1 \mathrm{mAh} \mathrm{cm}^{-2}$. 


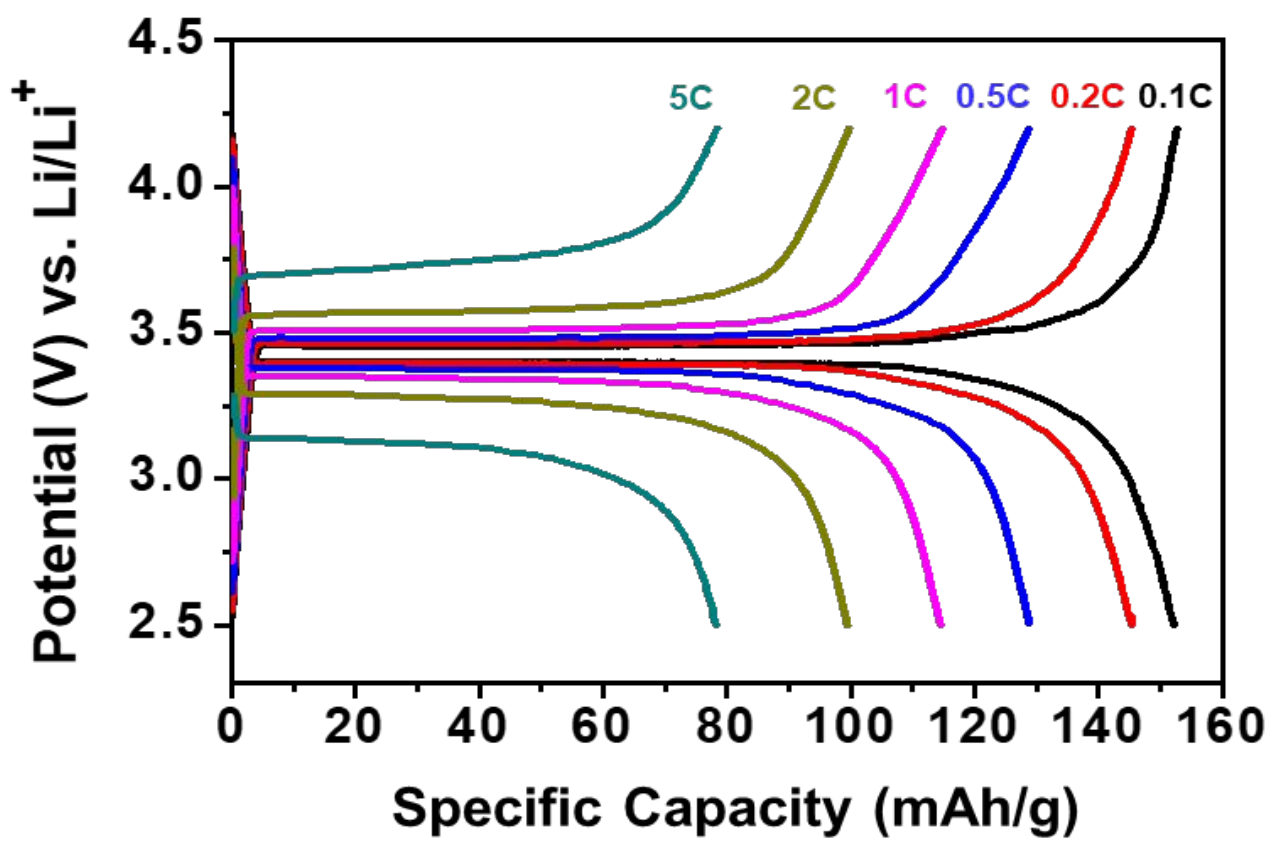

Figure S9. Charge-discharge profiles of LFP/40-INSPE 1:2/Li in coincell tested at $0.1 \mathrm{C}$, $0.2 \mathrm{C}, 0.5 \mathrm{C}, 1 \mathrm{C}, 2 \mathrm{C}$, and $5 \mathrm{C}$ at room temperature. 
(a)

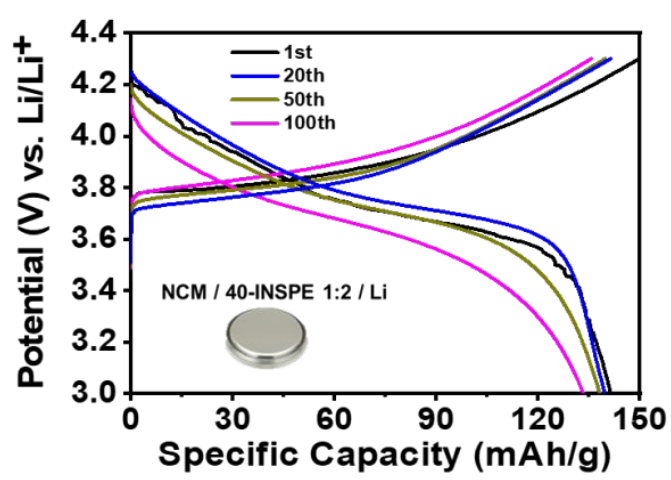

(b)

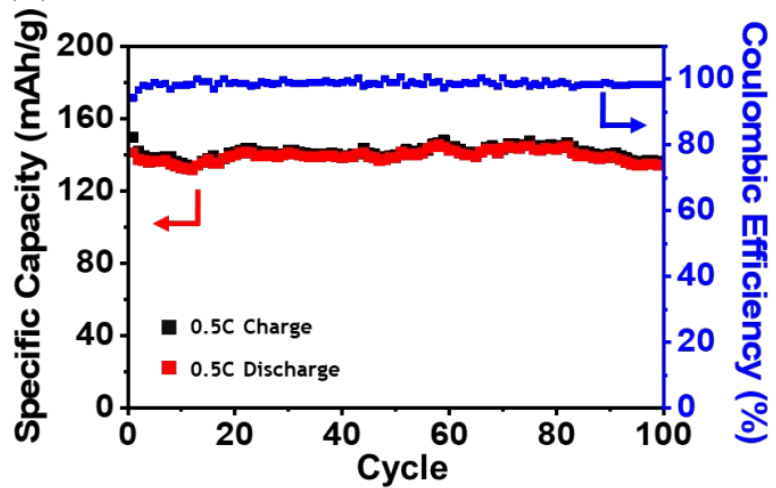

Figure S10. (a) Charge-discharge profiles, and (b) cyclic performance of NCM/40-INSPE 1:2/Li in coincell tested at 0.5 for 100 cycles. 
(a)

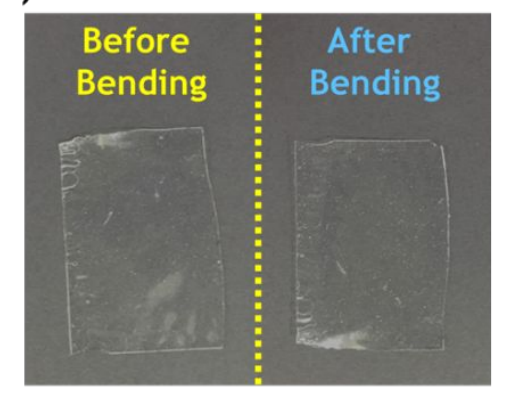

(b)

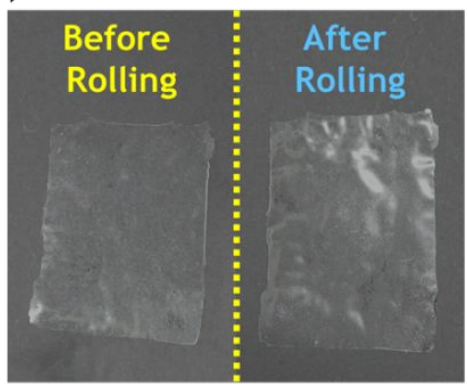

(c)

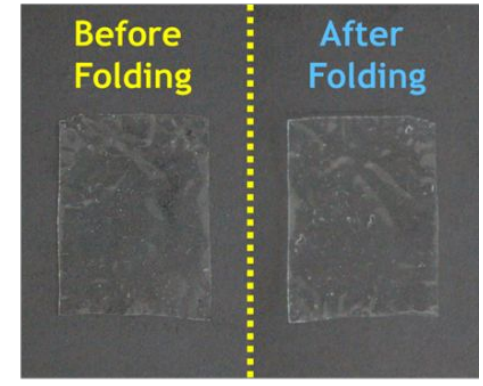

Figure S11. Comparison of 40-INSPE 1:2 before and after : a) bending (5000 cycles), b) rolling (5000 cycles), c) folding (2000 cycles). 
Video S1. LFP / 40-INSPE 1:2 / Li pouch-cell lighting up LEDs in letters under consecutive bending.

Video S2. LFP / 40-INSPE 1:2 / Li pouch-cell lighting up LEDs in letters under successive rolling.

Video S3. LFP / 40-INSPE 1:2 / Li pouch-cell lighting up LEDs in letters under double-folding. 